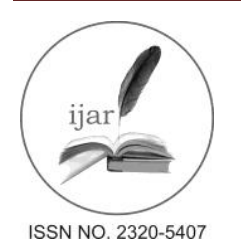

\author{
Journal homepage: http://www.journalijar.com

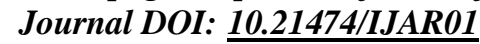

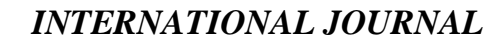

OF ADVANCED RESEARCH

RESEARCH ARTICLE

\title{
ACETYLSALICYLIC ACID (ASA) IMPAIRS INFLUENZA B VIRUS PROPAGATION AND DELAY APOPTOSIS IN INOCULATED A549 AND MDCK II CELL LINES.
}

\author{
Khalid Bassiouny $^{1}$, Ibrahim El-Sayed ${ }^{1}$, Aziz Nokaly ${ }^{2}$, Ahmed S. Abdelghani ${ }^{3}$, Wael Roshdy ${ }^{3}$. \\ 1. Genetic Engineering and Biotechnology Research Institute (GEBRI), University of Sadat City, Sadat City \\ 32897, Egypt. \\ 2. Faculty of Medicine, Al-Azhar University, Cairo 35527, Egypt. \\ 3. Central Public Health Laboratories (CPHL), Cairo 11613, Egypt.
}

\section{Manuscript Info \\ Manuscript History: \\ Received: 18 March 2016 \\ Final Accepted: 29 April 2016 \\ Published Online: May 2016}

Key words:

NF-kB ASA,

Influenza B Virus

TNF $\alpha$.

*Corresponding Author

Khalid Bassiouny.

\begin{abstract}
Despite the increasing knowledge in the influenza virus host interactions, most of the published work focuses on influenza A viruses, leaving a gap with respect to influenza $B$ virus host interactions, Here we examined acetylsalicylic acid (ASA), also known as aspirin, on A549 and MDCKII cells infected by INF B Yamagata virus, and the anti-NF-kB and antiinfluenza virus action of ASA were studied, our results show that a few millimolar concentration range from ASA which do not affect the viability of cultured cells, can impaired INF B virus propapagation and playing a role in delaying apoptosis via impaired $\mathrm{TNF} \alpha$ induced NF- $\mathrm{KB}$ signaling.
\end{abstract}

Copy Right,IJAR, 2016...All rights reserved.

\section{Introduction:-}

Despite the increasing knowledge in the influenza virus host interactions, most of the published work focuses on influenza A viruses, leaving a gap with respect to influenza B virus host interactions (B.-J. Kroesen et al., 2015; Ibrahim El-Sayed et al., 2016). Fatal influenza outcomes result from a combination of rapid virus replication and collateral lung tissue damage caused by exaggerated pro-inflammatory host immune cell responses. There are few therapeutic agents that target both biological processes for the attenuation of influenza-induced lung pathology (Jianxin Chen et al., 2015). They are enveloped; negative single stranded RNA viruses with a segmented genome divided into 8 genes that code for 11 proteins (B.-J. Kroesen et al., 2015) that not only act as viral components but also interact with the pathways of host infected cells, mainly to counteract the antiviral cell response and help the viral replication (R. Konig et al.,2010 ; P. Gaur et al.2011). Apoptosis induced during influenza virus infection is a major contributing factor to cell death and tissue damage (L. A. Santos et al., 2015).

All of the mammalian, as well as all of the avian, influenza viruses tested induce apoptosis in MDCK cells, which prove that apoptosis is a general mechanism by which influenza (Ludwig et al.,2006; Ibrahim El-Sayed et al., 2016). To date, up to 1023 interactions between viral and host proteins have already been described (Ibrahim ElSayed et al., 2016). (Ludwig et al., 2006; Ibrahim El-Sayed et al., 2016). One of the hallmark cellular responses to influenza virus infection is the activation of transcription factor NF-KB signaling (Ludwig et al., 2003; Ludwig et al., 2006; Mazur et al., 2007). Acetylsalicylic acid (ASA), also known as aspirin displays, at millimolar concentrations, several mechanisms independent from its ability to inhibit cyclooxygenases and inhibition of the activation of nuclear factor-nB by prevention of the phosphorylation and degradation of the inhibitory subunit IкB(Antonella et al.,2006). 
Acetylsalicylic acid (ASA), also known as aspirin, is an efficient and quite selective inhibitor of the NF-kBactivating kinase IKK2. Correspondingly, ASA efficiently blocked replication of influenza viruses, including H5N1 strains, in cell culture by several orders of magnitude in a concentration range that was not toxic for host cells (S. Ludwig 2009). The most crucial step in the canonical NF-kB pathway is the activation of the inhibitor of $\mathrm{kB}$ (IkB) kinase (IKK) that in turn phosphorylates IkB leading to its ubiquitinylation. IkB is then rapidly degraded and releases the transcriptionally active NF-kB factors, such as p50 or p65/A-Rel to migrate to the nucleus (Karin, 1999a). Among the three isozymes in the IKK complex IKKb, also named IKK2, is the enzymatically most important subunit towards phosphorylation of IkB (Karin, 1999b). Our study is based on the observation that ASA is a strong inhibitor of NF-KB signaling and may therefore impact upon influenza B propagation. We demonstrated that treatment of cells with ASA greatly reduced the yield of influenza B virus at sub-cytotoxic doses. Preincubation of virus with ASA pronouncedly inhibited influenza B virus plaque formation. Thus, in addition to its potential effects on cellular function, ASA also acts through direct interaction with viral particles that interrupts an early stage(s) of I influenza B infection. In addition, we confirmed that the effect of ASA on delaying intrinsic and extrinsic viral apoptosis and evaluated whether this effect is closely linked to NF-kB/IkB $\alpha$ regulation in the studied cell lines. First, we found that a low dose of ASA downstream regulation of TNF $\alpha$ and caspases 3 , caspases 8 , caspase 9 ,proapoptotic bad gene, interferon family MxA gene mRNA and protein expression. Second, ASA pretreatment inhibited TNF $\alpha$ induced activation of NF-kB by preventing the degradation of IkB $\alpha$. Finally, TNF $\alpha$ induced activation of endogenous IKK and subsequent phosphorylations of IkB $\alpha$ ware suppressed by ASA pretreatment. These observations suggest that the anti-inflammatory effect of ASA in lung epithelial cells may be due to the blocking of IkB $\alpha$ phosphorylation by suppressing IKK activity, thereby inhibiting subsequent degradation of $\mathrm{IkB} \alpha$, activation of NF-kB, and proinflammatory cytokine expression.

\section{Material and Methods:-}

\section{Cells:-}

The IFN competent human cells include alveolar epithelial cell line A549 and were obtained from central public health laboratories in Egypt(CPHL), Madin Darby canine kidney cells (MDCK II), European Collection of Cell Cultures (ECACC), London, UK), recommended by World Health Organization for influenza virus replication and were obtained from central public health laboratories in Egypt(CPHL), were kept in maintenance medium containing Dulbecco's Modified Eagle Medium , (DMEM; Gibco, Paisley, UK), 2 mM 1-Glutamine (Gibco),1× NEAA (Non-Essential Amino acids, Gibco), and $24 \mathrm{mM}$ HEPES(N-2-hydroxyethylpiperazine-N-2-ethane sulfonic acid, Gibco) at $30^{\circ} \mathrm{C}$. MDCK II cells were subcultured at 3-4 $310^{4}$ cells/cm2 with growth medium (maintenance medium supplemented with $10 \%$ fetal bovine serum (FBS; Gibco). Growth medium was supplemented with 1 $\mathrm{mg} / \mathrm{ml}$ Geneticin ${ }^{\circledR}\left(\right.$ Gibco) for cell line continuation or with $2.5 \mu \mathrm{g} / \mathrm{ml}$ Fungizone ${ }^{(G i b c o)}$ and $1 \times$ PSN (PenicillinStreptomycin-Neomycin Antibiotic Mixture, Gibco) when cells were plated to be infected. Cultures were incubated at $37^{\circ} \mathrm{C}$ for $48 \mathrm{~h}$ prior to infection to reach a minimum of $90 \%$ mono-layer confluence.

\section{Viruses:-}

Influenza B/Yamgata isolated in CPHL in Egypt, virus were propagated on MDCKII cells for stock constitution. Briefly, MDCK II cells in monolayer were infected with the virus; left to stand for $30 \mathrm{~min}$ for virus adsorption was as controls. Monolayer of the cells at a concentration of $1 \times 10^{6}$ cells $/ \mathrm{mL}$ were infected with the virus at a multiplicity of infection (MOI) $1.0 \mathrm{PFU} /$ cell in the presence of supplemental trypsin. Following adsorption for $1 \mathrm{~h}$ at $37^{\circ} \mathrm{C}$, the inoculum was removed and washed before DMEM replaced.

The cultures were incubated up to 48 hours post infection (hpi) and observed by inverted light microscopy for cytopathic effect (CPE). For each cell, four different sets of tissue culture flask were infected. Mock Infected cells served were titrated before and after infection as described previously (S. Shahsavandi (2013)).

\section{Cell Viability Assay:-}

A549 and MDCK cells were infected by Influenza B virus at MOI of 1.0 PFU/cell and culture supernatants obtained at $8 \mathrm{~h}$ intervals up to $48 \mathrm{hpi}$. Cell viability following viral infection was determined by 3-(4, 5-dimethylthiazol-2-yl)2, 5-diphenyl tetrazolium bromide (MTT) assay. In this case, each cell seeded in 96-well culture plates (approximately 5,000 cells per well) incubated with the related culture supernatants The cells were washed with PBS and incubated with DMEM and $50 \mu \mathrm{L} /$ well of MTT solution $(5 \mathrm{mg} / \mathrm{mL})$ for another $3 \mathrm{~h}$ at $37^{\circ} \mathrm{C}$. Then, the medium was totally removed, and $200 \mu \mathrm{L}$ of $0.04 \mathrm{~N} \mathrm{HCl}$ in isopropanol was added to each well, and the plate was incubated 
for $1 \mathrm{~h}$ at room temperature. Optical density value was measured at $540 \mathrm{~nm}$ using an ELISA reader. Cell survival was expressed as the ratio of virus infected to uninfected control. The three independent experiments were performed.

\section{LDH assay:-}

The media of infected cells were removed from each condition at 8, 24, 36 and $48 \mathrm{~h}$ post infection (hpi) and centrifuged at $250 \times \mathrm{g}$ for $4 \mathrm{~min}$. Total cell death was determined by measuring the release of LDH from cells with the CytoTox96®Non-Radioactive Cytotoxicity Assay kit (Promega, Madison, IL, USA), according to the manufacturer's instructions. The cell monolayer was recovered for morphological analyses of apoptosis.

\section{Compounds:-}

ASA, obtained from Sigma-Aldrich, was dissolved in DMSO at a stock concentration of $100 \mathrm{mM}$ and stored at $80^{\circ} \mathrm{C}$.

\section{Viral infections and ASA treatment:-}

MDCK cells $(4 \times 10$ 4/well) were seeded in 48 -well plates $16 \mathrm{~h}$ before infection. Cell monolayers were infected with 1.0 MOI pfu/cell (plaque forming units) of INF B virus. Supernatant from infected cells was collected at 12, 18, 24, and $30 \mathrm{~h}$ post-infection (hpi) and the yield of virus progeny was measured by plaque assay. For time of addition experiments, the indicated concentrations of ASA or mock treatment (DMSO) were added to the medium at various times of infection. Briefly, (1) pre-treatment: ASA was included in the cell culture medium for $8 \mathrm{~h}$ and was removed prior to virus infection; (2) simultaneous: ASA mixed with virus in the infection medium was added simultaneously to the cells and left on the cells throughout; (3) post-infection: ASA was added to cells at 2 hpi and remained throughout the time of infection.

\section{Plaque assay:-}

MDCK cell monolayers in 12 -well plates $(2 \times 104$ cells/well $)$ were washed twice with PBS followed by infection with serial dilutions of virus. After $2 \mathrm{~h}$ absorption at $37 \mathrm{C}^{\mathrm{C}}$, unbound viruses were removed and cells were then cultured for 2 days with $1 \mathrm{ml} /$ well MEM supplemented with $0.6 \%$ agarose at 37 _ and 5\% CO2. Viral plaques were visualized by staining with Giemsa (Sigma, St. Louis, MO).

\section{Cell immunofluorescence studies:-}

A549 cells grown on $15 \mathrm{~mm}$ cover slips (Mat Tek, USA) were infected with INF B virus at $1.0 \mathrm{MOI}$ for $1 \mathrm{~h}$ and treated with ASA or media control. At indicated time points, cells were fixed with $4 \%$ paraformaldehyde (PFA) in PBS for $10 \mathrm{~min}$, permeabilized with $0.25 \%$ Triton-X100 for $15 \mathrm{~min}$, then either stained with a mouse monoclonal antibody against the influenza A virus nucleoprotein (NP) (clone \#65, Immune Technology Corp, USA) or a rabbit monoclonal antibody against NF- $\mathrm{B}$ p65 (D14E12) (Cell Signaling Technology Co., USA) in 1:200 dilution at $4{ }^{\circ} \mathrm{C}$ for $1 \mathrm{~h}$. Cells were then stained with Alexa Fluor 488 conjugated goat anti-mouse IgG (Cell Signaling Technology Co., USA, 1:1000 dilutions). Cell nuclei were stained using 4', 6-diamidino-2-phenylindole (DAPI: $1 \mu \mathrm{g} / \mathrm{ml}$ ) (Sigma Chemical Co., USA). Cells Stained cells were observed with a fluorescence microscope (Olympus BX51).

\section{Real Time PCR Analysis:-}

Total RNA was extracted from cells using (Qiagen) according to the manufacturer's protocol. Five hundred nanograms of purified mRNA were used to generate cDNA with random hexamer primers (Thermo Scientific) and Revert Aid H Minus M-MuL V Reverse Transcriptase (Thermo Scientific) according to the manufacturer's protocol.

The quantitative real-time PCR (qRT-PCR) reaction mixture $(25 \mu \mathrm{l})$ consisted of the following: $12.5 \mu 1$ of Maxima SYBR green PCR master mix (Thermo Scientific), $0.5 \mu \mathrm{l}$ of cDNA template, and $1 \mu \mathrm{l}$ of each primer $(100 \mu \mathrm{M}$ forward and reverse primers) (Table 1). Reactions were run in duplicate on Applied Biosystems 7500 real-time PCR system. The cycling conditions were as follows: $2 \mathrm{~min}$ at $50^{\circ} \mathrm{C}, 2 \mathrm{~min}$ at $95^{\circ} \mathrm{C}$, and 50 cycles, with 1 cycle consisting of $15 \mathrm{~s}$ at $95^{\circ} \mathrm{C}$ and $30 \mathrm{~s}$ at $60^{\circ} \mathrm{C}$. Threshold cycle (CT) values were normalized to the values for the GAPDH control and compared $\beta$-actin controls Primers for real-time PCR of RNA transcripts. 
Table 1: Ibrahim El-sayed et al., 2016.

\begin{tabular}{|c|c|c|}
\hline Name & DIRECTION & Sequence $5^{\prime} \rightarrow 3^{\prime}$ \\
\hline MxAF & forward primer, & TTCAGCACCTGATGGCCTATC \\
\hline MxAR & reverse primer & TGGATGATCAAAGGGATGTGG \\
\hline b-actin & forward primer & GAG ACC TTC AAC ACC CCG C \\
\hline b-actin & reverse primer & ATG TCA CGC ACG ATT TCC C \\
\hline BAD & forward primer & ACCCGGCAGACAGATGAG \\
\hline $\mathrm{BAD}$ & reverse primer & CTTCCTCTCCCACCGTAGC \\
\hline $\mathrm{I} \kappa \mathrm{B} \alpha$ & forward primer & CAGCAGACTCCACTCCACTT \\
\hline $\mathrm{I} \kappa \mathrm{B} \alpha$ & reverse primer & GAGAGGGGTATTTCCTCGAA \\
\hline TNF- $\alpha$ & forward primer & CTCTTCTCCTTCCTGATCGTGGCA \\
\hline TNF- $\alpha$ & reverse primer & GTTGGATGTTCGTCCTCCTCACA \\
\hline CASPASE 3 & forward primer & TTAATAAAGGTATCCATGGAGAACACT \\
\hline CASPASE 3 & reverse primer & TTAGTGATAAAAATAGAGTTCTTTTGTGAG \\
\hline CASPASE 9 & forward primer & AGCCAGATGCTGTCCCATAC \\
\hline CASPASE 9 & reverse primer & CAGGAGACAAAACCTGGGA \\
\hline CASPASE 8 & forward primer & CTGGGAAGGATCGACGATTA \\
\hline CASPASE 8 & reverse primer & CATGTCCTGCATTTTGATGG \\
\hline GAPDH & forward primer & GGCATTGCTCTCAATGACAA \\
\hline GAPDH & reverse primer & TGTGAGGGAGATGCTCAGTG \\
\hline
\end{tabular}

\section{Result and Discussion:-}

ASA inhibits INF B replication in A549 and MDCK II cells;-

ASA cytotoxicity was first established using the MTT viability assay on A549 and MDCK II cells (Figure 1). Minimal cytotoxicity was observed for ASA concentrations $7 \mathrm{mM}$ at $48 \mathrm{~h}$ post treatment on A549 cells in vitro (Figure 1) and $7 \mathrm{mM}$ ASA hence selected as the maximal drug concentration used for subsequent INF B infection studies. ASA downregulates TNF $\alpha$-induced NF- $\kappa$ B signaling (CHUL et al.,2001). To investigate for potential in vitro anti-INF B activity, we first evaluated the effects of ASA on INF B propagation in A549 cells and MDCK II. A549 cells were infected with INF B Yamagata with MOI 1.0 for 8, 24, 48 and 72 h post-infection and total cell and supernatant virus titres calculated. Acetylsalicylic acid (ASA; aspirin) has been reported to inhibit in vitro and in vivo influenza virus viral replication through down regulation of $\mathrm{NF}-\kappa \mathrm{B}$ signaling (CHUL et al.,2001) and Amantadine was used as a positive control. IC50 values for the inhibition of INF B replication were $>10 \mathrm{mM}$ of ASA. For INF B Yamagata, ASA inhibited INF B replication in a dose-dependent manner from 8, 24, 48 and 72 h post-infection to the inhibitory effects of ASA (Figure 1).

1) A549

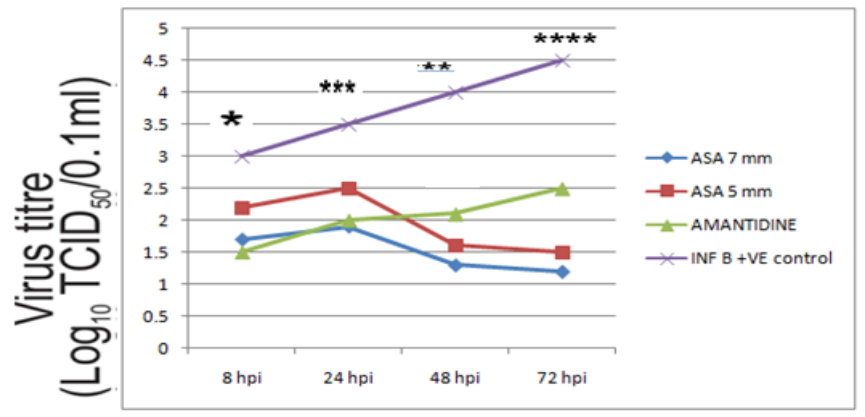

Time post infection

Figure1: ASA attenuates INF B replication in A549 cells. A. INF B Yamagata at 100 TCID50 for $2 \mathrm{~h}$ and then incubated with 7 or $5 \mathrm{mM}$ ASA or Amantadine respectively. Cells and supernatants were harvested at 8, 24, 48 and $72 \mathrm{~h}$ post-infection and total INF B yield calculated by TCID50 titration using MDCK cells. Data represented as mean \pm SEM of three independent experiments. $* \mathrm{P}<0.05, * * \mathrm{P}<0.01, * * * \mathrm{P}<0.001, * * * * \mathrm{P}<0.0001$. 


\section{ASA affects an early stage of virus infection:-}

Time of addition experiments was performed to determine the stage(s) at which ASA exerted its inhibitory effects. ASA was added to MDCK cells at three distinct time points: prior to infection (pre-treatment), at the same time as virus infection (simultaneously), or at $2 \mathrm{hpi} \mathrm{(after} \mathrm{entry).} \mathrm{As} \mathrm{shown} \mathrm{in} \mathrm{Fig.} \mathrm{2,} \mathrm{MDCK} \mathrm{cells} \mathrm{pre-treated} \mathrm{with} \mathrm{ASA} 7$ $\mathrm{mM} 8 \mathrm{~h}$ prior to infection (but removed just before virus infection) reduced the production of virus to $20 \%$ at 12,18 , and $24 \mathrm{hpi}$ (through effects on NF-кB). The addition of ASA $7 \mathrm{~mm}$ simultaneously with virus resulted in a much stronger inhibition than that of cells pre-treated with ASA $7 \mathrm{mM}$ at 18 and $24 \mathrm{hpi}$ (Fig. 2, with significance p < 0.05). Importantly, addition of ASA $7 \mathrm{~mm}$ at $2 \mathrm{~h}$ after infection reduced the degree of inhibition (in the case of the 18 and $24 \mathrm{~h}$ time points back to the pre-treatment levels). This suggested that ASA $7 \mathrm{mM}$ may directly interfere with a very early stage (possibly directly with the virus particle), to prevent infection. We therefore performed plaque reduction assays to measure the plaque formation ability of INF B particles pre-incubated with ASA $7 \mathrm{mM}$. As indicated in Fig.3, the minimal concentration for complete inhibition was $7 \mathrm{~mm}$ (Fig. 3) and the EC50 (i.e. The concentration of ASA that reduced the plaque formation by 50\%, relative to the control without test compound) was $10 \mathrm{~mm}$. Given the CC50 of ASA was $10 \mathrm{~mm}$, the selectivity index (SI) value (CC50/EC50) of ASA $7 \mathrm{~mm}$ is approximately 92.5, higher than several anti-influenza agents published elsewhere (Songet al., 2007). Since the inhibitory effect was observed when virions were pre exposed to ASA $7 \mathrm{mM}$ prior to infection, whereas when it was introduced into the cell culture medium after virus attachment, a moderate inhibitory effect was observed in the yield of progeny viruses (Fig. 3).

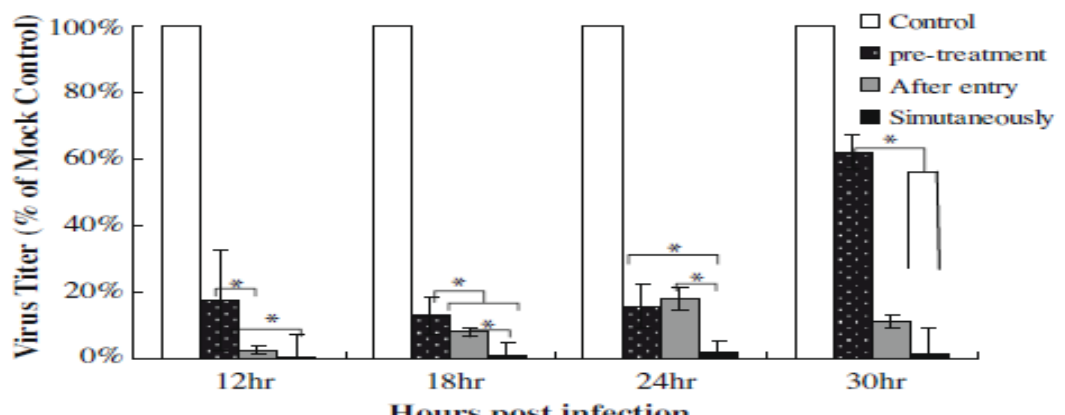

Fig. 2:- The effect of ASA on different stages of virus infection. About $7 \mathrm{mM}$ curcumin was added to cells at three distinct time points: 8-h prior to infection (pre-treatment), at the same time as virus infection (simultaneously), or at $2 \mathrm{hpi}$ (after entry). The yield of progeny viruses in supernatant was determined at 12,18, 24, and $30 \mathrm{hpi}$ * indicates the $\mathrm{p}$-value $<0.05$.

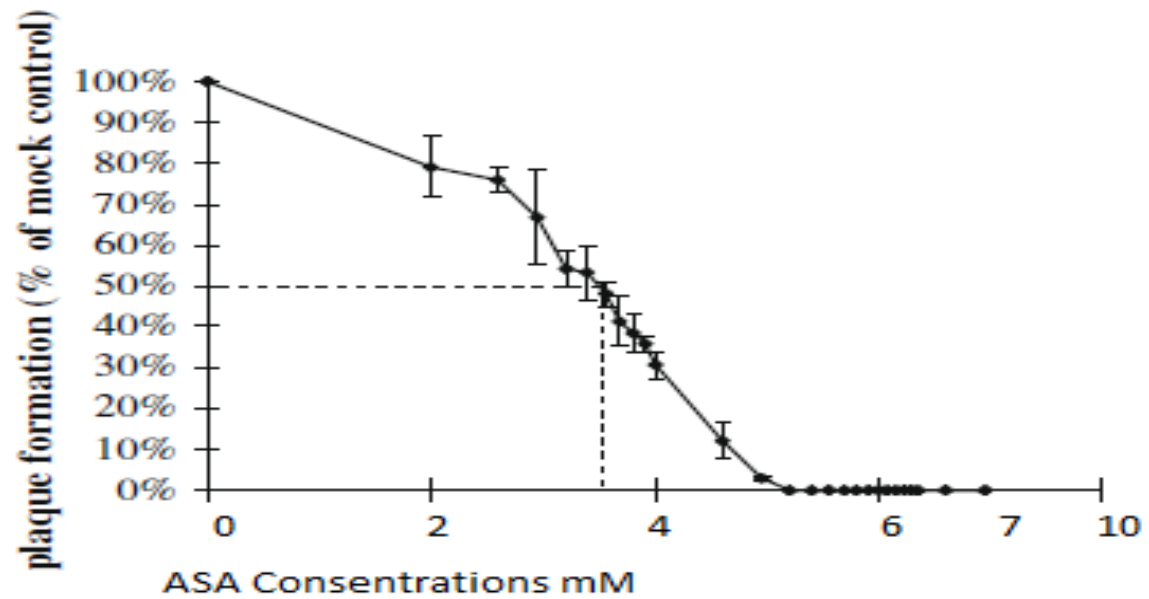

Fig. 3:- ASA reduces plaque formation activity. Data are from three independent experiments. The dose-dependent effect of ASA treatment was observed and the dotted line shows the EC50 of $10 \mathrm{mM}$. 


\section{ASA suppresses INF B-induced NF- $\kappa B$ activation in A549 and MDCK II cells:-}

ASA suppresses I $\mathrm{K} B \alpha$ degradation in a dose dependent manner. Because NF-kB exists as an inactive form bound to the inhibitory protein $I \kappa B \alpha$ in the cytoplasm, the degradation of $I \kappa B \alpha$ must occur in order for NF-kB to translocate to the nucleus (Xunlong Shi 2013). We next analyzed the effect of ASA on TNF- $\alpha$ induced degradation of IkB- $\alpha$.

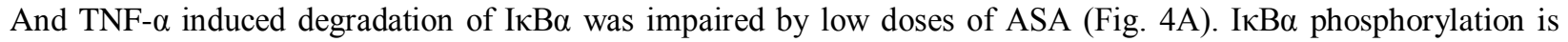
blocked by ASA. IкB $\alpha$ degradation by a proteasome dependent pathway is preceded by phosphorylation on two serine residues (Ser32 and Ser36) and ubiquitination (Naveen et al.,2008. To address the mechanism involved in the

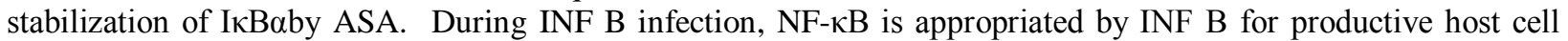
infection and active NF- $\kappa B$ signaling is required for INF B propagation itself (Nimmerjahn, F. 2004). To investigate whether ASA mediated inhibition of INF B replication was dependent on NF- $\kappa$ B signaling in INF B Yamagata virus infections, we examined the effects of ASA treatment on NF- $\mathrm{KB}$ nuclear translocation in INF B infected A549 cells. At steady state, NF- $\mathrm{KB}$ predominantly exists as a heterodimer of RelA (p50) and p65 subunits bound by an

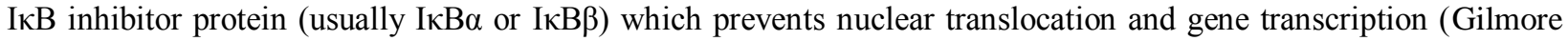

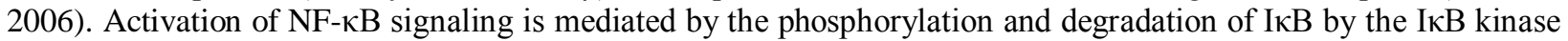
complex, facilitating nuclear translocation of RelA/p65 subunits where they bind to NF- $\mathrm{kB}$ promoter regions and initiate gene transcription (J.M. Hill et al., 2015.). As expected, INF B infection greatly increased nuclear but not cytosol p65 levels at $24 \mathrm{~h}$ post-infection compared to non-infected controls, demonstrating INFB induced activation of NF- $\mathrm{KB}$ signaling, ASA treatment attenuated INF B-induced nuclear p65 translocation (Figure 4b). Consistent with attenuated p65 nuclear translocation, ASA treatment also decreased the level of INFB induced IкB $\alpha$ degradation in infected A549 cells (Figure 2A and 2B). Immunofluorescence studies further confirmed decreased p65 nuclear translocation in ASA treated INFB infected A549 cells at $8 \mathrm{~h}$ post-infection compared to untreated controls (Figure 4b). ASA addition itself did not affect p65 nuclear translocation similar to non-infected (mock) A549 cells (Figure 4b) although a modest but statistically significant increase in cytosolic p65 expression was observed at $7 \mathrm{mM}$.

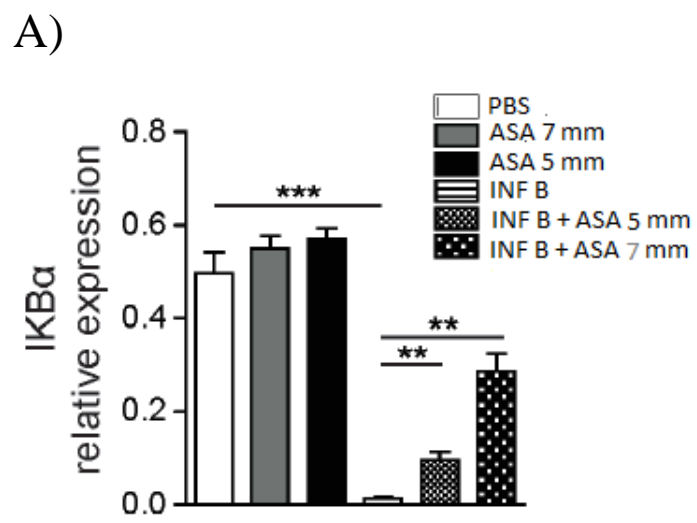


B)

DAPI
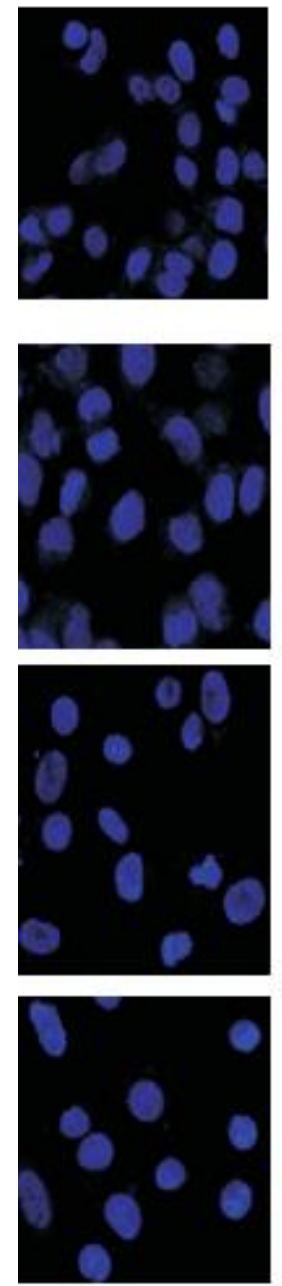

P65
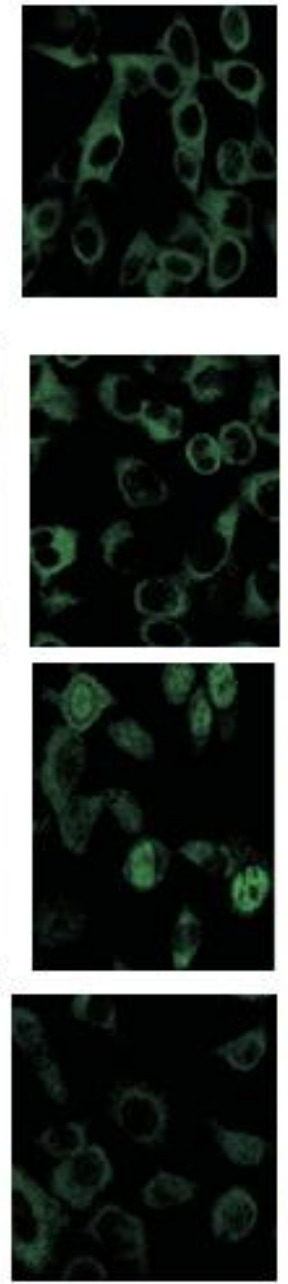

Merge

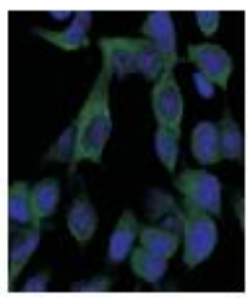

Mock
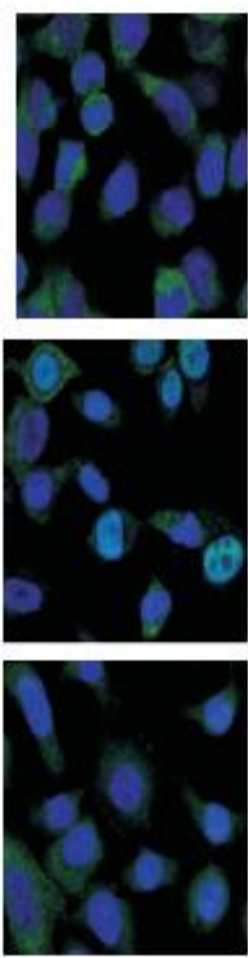

Mock + ASA $7 \mathrm{~mm}$

VIRUS

Mock

VIRUS + ASA $7 \mathrm{~mm}$

Figure 4: ASA inhibits nuclear NF- $\kappa B$ protein translocation in INF B infected A549 cells A- Relative gray scale values of total protein I $\kappa \mathrm{B} \alpha$ expression quantified from $\mathrm{C}$. $* \mathrm{P}<0.05$. B. INF B infected A549 cells $(\mathrm{MOI}=1,1 \mathrm{~h})$ were cultured $\pm 7 \mathrm{mM}$ for $8 \mathrm{~h}$ post-infection, fixed and stained for p65 expression (p65: green, DAPI nuclear stain: blue, scale $=10 \mu \mathrm{m}$ ). Representative of three independent experiments. Non-infected $\pm 7 \mathrm{mM}$ SSa treated A549 cells were visualised in parallel.

ASA delay apoptotic signaling pathways idused by INF B virus on MDCK II and A549 cells:-

A number of studies have reported the important role apoptosis plays in promoting efficient influenza virus replication (Z. Xing et al.,2010). INF B virus infected A549 cell and treated with ASA $7 \mathrm{mM}$ also elicited less TNF $\alpha$ and bad transcription than either or mock or infected cells alone, These observations can account for the lower apoptotic response in influenza B virus infected lung (Figure 5), INF B prefers extrinsic pathway in A549 cells according to significant low or undetectable caspase 9 activity and high activity of caspase 8 but also can induce intrinsic pathway in MDCK II cells as determined by significant low or undetectable activity of caspase 8 and high caspase 9 activity at different MOIs (Ibrahim El-sayed et al.,2016). The delayed onset of cytopathogenicity of INF B by ASA ,by which ASA down regulate caspases 3, 8,9 mRNA, proapoptotic bad ,TNF $\alpha$,IKB $\alpha$ and MXA expressions, We focused mainly on the expression profiles of these genes that revealed a significant infection effect with an absolute $\log$ fold change relative to untreated INF B at any time-point of ASA treatment (referred to as differentially expressed genes) as shown in Fig.5 The most striking result was that A549 and MDCK II cells responded to ASA $7 \mathrm{mM}$ more rapidly, all studied genes were down regulated as early as $16 \mathrm{hpi}$, whereas their 
down expression was observed at 16,24,36 and 48 hpi. Interestingly, the maximal expression changes for most genes in all infections were quite comparable. Notably, more intense expression changes in response to ASA coincided with relatively higher viral protein synthesis detected at 16 and $48 \mathrm{hpi}$ as described earlier. We previously demonstrated that $\mathrm{A}$ dose and time dependent activation of caspase 3, caspase 8, and caspase 9 proteins was observed in INFB infected A549 and MDCK II cells and INF B can induce extrinsic apoptosis pathway in A549 cells while it induce intrinsic pathway in MDCK II cells according to capases 8,9 To determine whether ASA has effect on the death receptor or mitochondrial pathways modulated apoptosis, samples from the infected cells were analyzed for the levels of expressions of proapoptotic marker bad and caspase 3, caspase 8 , and caspase 9 using quantitative RT-PCR The levels of caspase 8 protein activation were not detected in INF B infected MDCK II cells (Fig 5); however, the cell viability was significantly lower in MDCK II than A549-infected cells (Figure 5). The apoptotic markers in A549 and MDCK II cells were evaluated by quantitative RT-PCR (Table 1). However, different regulation levels of MxA mRNA were observed in A549 and MDCK II cells infected by INF B, and expression of MxA gene was upregulated at 16 hpi which reached to maximal level at 24 hpi compared with mock cells; however, the higher dose of virus is, a weaker MxA expression was detected. INF B induces an immediate apoptotic response, but INF B induced cell death levels are depending on the type of cells (Figure 6).

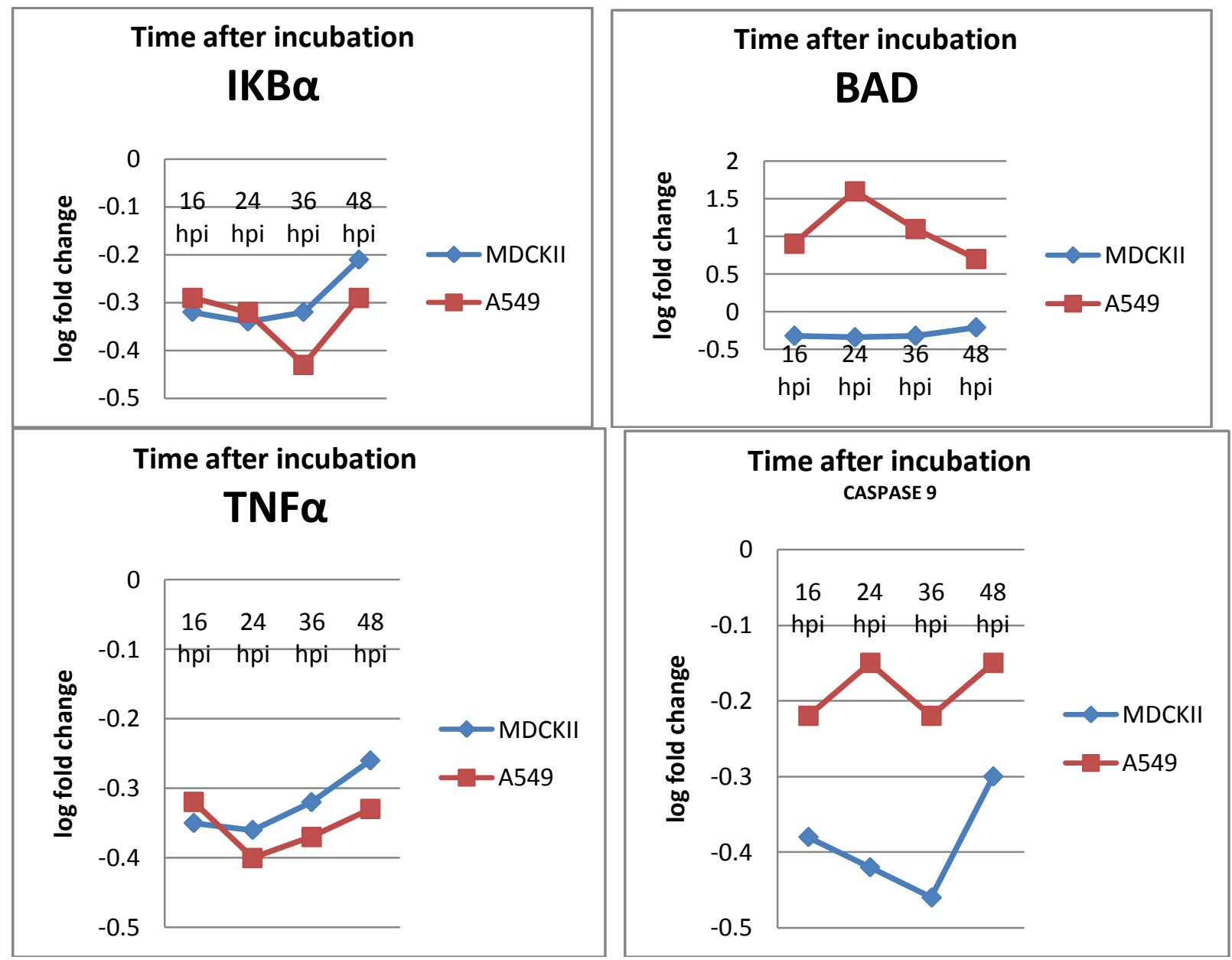



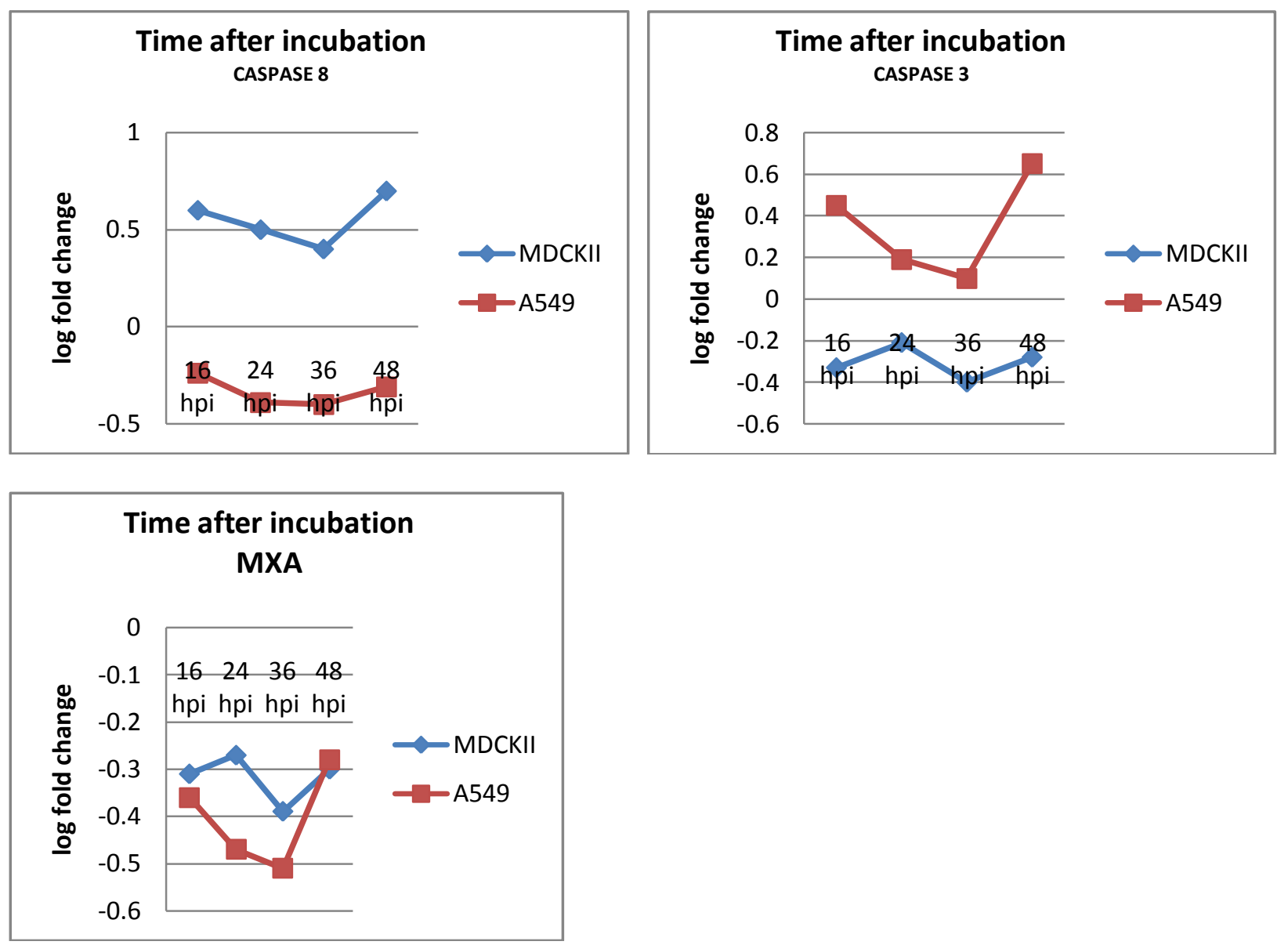

Figure 5: Validation of data for several apoptotic and cellular defense related genes by real-time RT-PCR. Log fold changes (relative to untreated A549 AND MDCKII cells) shown in the graphs reflect the average of duplicate samples.

6)

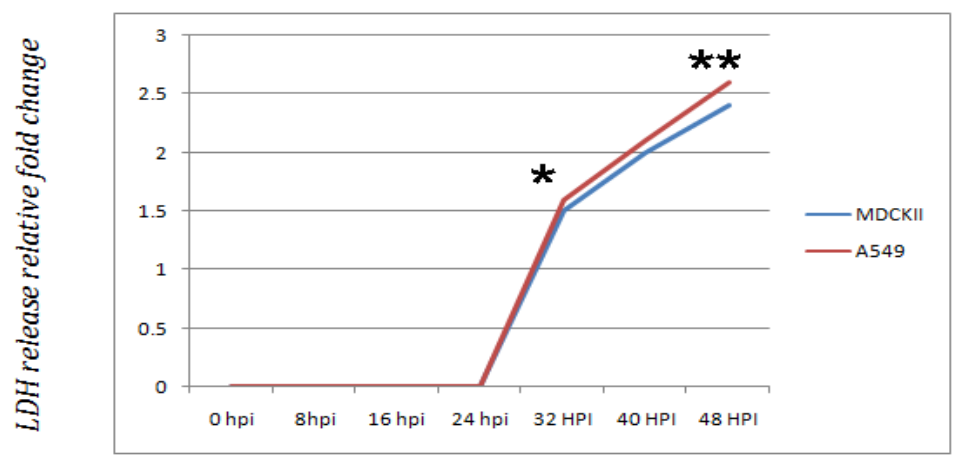

Time post infection

Figure 6: Flu B/Yamagata viruses induce cell death and apoptosis profiles. Effects of influenza infection (MOI 1.0) on viability of MDCK II (a), and A549 cells (b) were determined by LDH assay. The * indicates significant differences $(\mathrm{P}<0.05)$ and $* * \mathrm{P}<0.01$ from the mock cells at the same time after infection. 
Blocking TNF- $\alpha$ by using etanercept suppressed the immunopathology and mortality in lethal influenza-infected mice. These effects may be ascribed to the inhibition of the cytokine bursts, reduced inflammatory cell infiltration, and downregulation of NF- $\mathrm{NB}$ signaling pathways, this is the first attempt at etanercept use in influenza virusinduced viral pneumonia, and more details must be clarified, such as the influences of IFN system, adaptive immune responses, and different virus strains. ASA prevents MTX-mediated apoptosis via inhibition of caspase-3 activation and upregulation of Bcl-2 expression (KUN-HUANG YAN et al., 2013). We next examined the effects of ASA treatments on MDCK II and A549 cells and determined apoptosis after 16,24,36 and 48 hpi where bad gene BCL-2 family is upregulted on A549 cells were it downregulated in MDCKII cells (Fig. 5). Importantly, the number of apoptotic markers MDCKII and A549 cells was significantly reduced by ASA (Fig. 5).

\section{Conclusions:-}

Influenza viruses afflict millions of people each year and cause serious medical complications. Because of the high genetic variability of influenza viruses, the development of effective vaccines against pandemic influenza is still an ongoing challenge. The anti-NF-kB and anti-influenza virus action of ASA in cell culture only occurs in a millimolar concentration range which does not affect the viability of cultured cells, and playing a role in delaying apoptosis via impaired $\mathrm{TNF} \alpha$ induced NF- $\mathrm{NB}$ signaling.

\section{Conflict of Interests:-}

The authors declare that there is no conflict of interests regarding the publication of this paper.

\section{Acknowledgments:-}

1. The authors would like to thank Dr. Amel Nagiub, Ph.D., Head of the Virology Departments, Central Public Health Labs (CPHL), for her efforts and continues support.

2. Dr. Amer Sayed Ahmed and Dr. Heba Sami in Molecular Biology and Virus Isolation units in CPHL for their great efforts and for viruses and cell lines supports.

\section{References:-}

1. Antonella di Palma, Giuseppe Matarese, Vincenza Leone, Tiziana Di Matola, Fabio Acquaviva, Angela Maria Acquaviva, and Paolo Ricchi.(2006). Aspirin reduces the outcome of anticancer therapy in Meth Abearing mice through activation of AKT-glycogen synthase kinase signaling. Molecular Cancer Therapeutics. 5(5):1318-1324.

2. B.-J. Kroesen, N. Teteloshvili, K. Smigielska-Czepiel et al.,(2015).Immuno-miRs: critical regulators of T-cell development, function and ageing. Immunology. 144( 1): 1-10.

3. CHUL-GYU YOO, SEUNGHEE LEE, CHOON-TAEK LEE, YOUNG WHAN KIM, SUNG KOO HAN, AND YOUNG-SOO SHIM(2001). Effect of acetylsalicylic acid on endogenous IkB kinase activity in lung epithelial cells. Am J Physiol Lung Cell Mol Physiol 280: L3-L9.

4. Ibrahim El-Sayed, Khalid Bassiouny, Aziz Nokaly, Ahmed S. Abdelghani, and Wael Roshdy.(2016).Influenza A Virus and Influenza B Virus Can Induce Apoptosis via Intrinsic or Extrinsic Pathways and Also via NF-kB in a Time and Dose Dependent Manner. Biochemistry Research International. 2016:1-14.

5. Gilmore, T.D.,(2006). Introduction to NF-kappaB: players, pathways, perspectives. Oncogene. 25: 6680-6684.

6. Igor Mazur, Walter J. Wurzer, Christina Ehrhardt, Stephan Pleschka, Pilaipan Puthavathana, Tobias Silberzahn, Thorsten Wolff, Oliver Planz and Stephan Ludwig(2007). Cellular Microbiology (2007) .9(7): 1683-1694.

7. J.M. Hill, C. Clement, Y. Zhao, and W. J. Lukiw(2015).Induction of the pro-inflammatory NF-kB-sensitive miRNA-146a by human neurotrophic viruses.Frontiers in Microbiology. 6(43):1-4.

8. Jianxin Chen , Mubing Duan , Yaqin Zhao, Fangfang Ling, Kun Xiao, Qian Li , Bin Li, Chunni Lu, Wenbao Qi, Zhenling Zeng, Ming Liao, Yahong Liu and Weisan Chen.(2015). Saikosaponin A inhibits influenza A virus replication and lung immunopathology. Oncotarget.6 (40): 42541-42556.

9. Karin, M. (1999a) The beginning of the end: I kappa B kinase (IKK) and NF-kappa B activation. J Biol Chem 274: 27339-27342.

10. Karin, M. (1999b). How NF-kappaB is activated: the role of the IkappaB kinase (IKK) complex. Oncogene .18: 6867-6874. 
11. KUN-HUANG YAN, LIANG-MING LEE, MAO-CHIH HSIEH, MING-DE YAN,CHIH-JUNG YAO, PEY-YI CHANG, TSUNG-LI CHEN, HWAN-YOU CHANG, ANN-LII CHENG, GI-MING LAI and SHUANG-EN CHUANG.(2013) Aspirin antagonizes the cytotoxic effect of methotrexatein lung cancer cells. Oncology reports. 30: 1497-1505.

12. L. A. Santos, S. Sol'a, C. M. P. Rodrigues, and H. R. de Andrade (2015). Distinct kinetics and pathways of apoptosis in influenza A and B virus infection. Virus Research Journal. 205: 33-40.

13. Ludwig, S., Planz, O., Pleschka, S., \& Wolff, T. (2003). Influenza-virus-induced signalling cascades: Targets for antiviral therapy? Trends in Molecular Medicine, 9(2): 46-52.

14. Ludwig, S., Pleschka, S., Planz, O., \& Wolff, T. (2006). Ringing the alarm bells: Signalling and apoptosis in influenza virus infected cells. Cellular Microbiology, 8(3):375-386.

15. Naveen Kumar, Zhong-tao Xin, Yuhong Liang, Hinh Ly, and Yuying Liang(2008). NF-KB Signaling Differentially Regulates Influenza Virus RNA Synthesis. JOURNAL OF VIROLOGY.82(20):9880-9889.

16. Nimmerjahn, F. (2004). Active NF-kappaB signalling is a prerequisite for influenza virus infection. J Gen Virol.85: 2347-2356.

17. P. Gaur, A. Munjhal, and S. K. Lal, (2011).Influenza virus and cell signaling pathways. Medical Science Monitor. 17( 6) : RA148-RA154.

18. R. Konig, S. Stertz, Y. Zhou et al., (2010).Human host factors required for influenza virus replication. Nature. 463( 7282) : 813-817, 2010.

19. S. Shahsavandi, M. M. Ebrahimi,A.Mohammadi, andN. Zarrin Lebas(2013).Impact of chicken-origin cells on adaptation of a low pathogenic influenza virus. Cytotechnology..65( 3) : 419-424.

20. S. Ludwig (2009). Targeting cell signalling pathways to fight the flu: towards a paradigm change in antiinfluenza therapy. Journal of Antimicrobial Chemotherapy. 64(1): 1-4.

21. Xunlong Shi, Wei Zhou, Hai Huang, Hongguang Zhu, Pei Zhou, Haiyan Zhu and Dianwen Ju (2013). Inhibition of the inflammatory cytokine tumor necrosis factor-alpha with etanercept provides protection against lethal H1N1 influenza infection in mice. Critical Care.17:1-9.

22. Z. Xing, R. Harper, J. Anunciacion et al., (2010).Host immune and apoptotic responses to avian influenza virus H9N2 in human tracheobronchial epithelial cells. American Journal of Respiratory Cell and Molecular Biology. 44(1): 24-33. 